\title{
Efecto de suplementación con probiótico (lactobacillus) en dietas de alfalfa y concentrado sobre parámetros productivos de cuyes mejorados en crecimiento y engorde
}

\section{Effect of supplementation with probiotic (lactobacillus) in alfalfa and concentrate diets on productive parameters of improved guinea pigs in growth and fattening}

\author{
Canto Francys, Bernal Wilmer, ${ }^{2}$ José Saucedo ${ }^{3}$
}

\begin{abstract}
RESUMEN
El objetivo de la investigación fue evaluar el efecto de suplementación con probiótico (lactobacillus) en dietas de alfalfa y concentrado sobre parámetros productivos de cuyes mejorados en crecimiento y engorde. Se realizó en la estación experimental de animales menores de la Universidad Nacional Toribio Rodríguez de Mendoza de Amazonas. Se utilizaron 40 cuyes machos destetados a los $21 \pm 3$ días de edad con un peso inicial de $322 \mathrm{~g}$. La fase experimental duró 60 días. Se evaluaron cinco tratamientos: $\left(T_{1}\right.$, control), $T_{2}, T_{3}, T_{4}$ y $T, \quad(0.2,0.3,0.4$ y $0.5 \%$ probiótico, respectivamente). Con cuatro repeticiones por tratamiento ( 2 cuyes por repetición). Se usó un diseño completo al azar y para la comparación de medias la prueba de Tukey. No hubo diferencias significativas $(\mathrm{p}>0.05)$ para peso final, ganancia de peso diario, consumo de alimento diario, conversión alimenticia y rendimiento de carcasa. El T presentó mejor mérito económico, con $\mathrm{S} / 5.9$ por cuy logrado y un $26 \%$ mayor al T1. Los Probióticos como el lactobacillus se podría usar en la alimentación de cuyes hasta un nivel de $0.2 \%$ en la dieta, sin afectar los parámetros productivos, pero aportando un mejor mérito económico.
\end{abstract}

Palabras clave: alfalfa, indicadores productivos, Cavia porcellus, merito económico

\begin{abstract}
The objective of the research was to evaluate the e $\square$ ect of probiotic supplementation (lactobacillus) in alfalfa and concentrate diets on productive parameters of improved guinea pigs in growth and fattening. It was carried out in the experimental station of smaller animals of the UNTRM (Chachapoyas-Amazonas). Using 40 male guinea pigs weaned at $21 \pm 3$ days of age with an average initial weight of $322 \mathrm{~g}$. The experimental phase lasted 60 days. Five treatments were evaluated: ( $\mathrm{T}_{1}$, control), $\mathrm{T}_{2}, \mathrm{~T}_{3}, \mathrm{~T}_{4}$ and $\mathrm{T}_{s}(0.2,0.3,0.4$ and $0.5 \%$ probiotic, respectively). With four repetitions for treatment ( 2 guinea pigs for repetition). A complete random design was used and for the comparison of means the Tukey test. There were no significant differences $(p>0.05)$ for final weight, daily weight gain, daily feed intake, feed conversion and carcass yield. The $\mathrm{T}_{2}$ presented better economic merit, with $\mathrm{S} / 5.9$ per guinea pig achieved and $26 \%$ higher than $\mathrm{T}_{1}$. Probiotics such as lactobacillus could be used to feed guinea pigs up to a level of $0.2 \%$ in the diet, without att ecting the productive parameters, but providing a better economic merit.
\end{abstract}

Keywords: alfalfa, productive indicators, Cavia porcellus, economic merit

\footnotetext{
${ }^{1}$ Ingeniero zootecnista. Investigador del IGBI- UNTRM. Correo electrónico: fcantosaenz@gmail.com

${ }^{2}$ Ingeniero zootecnista. Docente FIZAB- UNTRM. Correo electrónico: wilmer.bernal@untrm.edu.pe

${ }^{3}$ Ingeniero zootecnista. Investigador del IGBI- UNTRM. Correo electrónico: saucedouriarte@gmail.com
} 


\section{INTRODUCCIÓN}

El cuy es un mamífero herbívoro que se alimenta principalmente de forraje verde, y según su anatomía gastrointestinal está clasificado como un fermentador post gástrico cecal (Van Soest, 1983). Es necesario conocer que la óptima digestión fermentativa depende del bienestar y equilibrio de la flora cecal, pues cualquier factor que la altere podría tener efectos desfavorables sobre el crecimiento, como por ejemplo, el número de bacterias presentes en el colon y la existencia de bacterias dominantes y subdominantes, ya que estas interacciones ocurren comúnmente, así como también, la competencia por nutrientes o la producción de moléculas antibióticas (Leandro y Victoria, 2012; Saavedra, 2018). Bourliux, et al., 2002).

Los probióticos están constituidos por dos o más microorganismos de bacterias acido lácticas y levaduras (García et al., 2002; Beasley et al., 2004; Mountzouris et al., 2007; Huertas, 2010). Estas, en el alimento, ejercen un efecto promotor de crecimiento, debido principalmente a la exclusión de microorganismos patógenos, efecto antimicrobiano y la producción de ácido láctico, lo que les permite crear un ambiente favorable para la respuesta inmunológica y la prevención de enfermedades infecciosas en los animales (Pascual et al., 1999; Bocourt et al., 2002 y Pérez et al., 2002; Mejia y Vanessa, 2011).

Estos probióticos se han considerado como una de las alternativas naturales al uso de antibióticos pues no generan efectos colaterales y producen mejor digestibilidad, ganancia en peso y mayor índice de conversión alimentaria (Gutiérrez, Montoya y Vélez, 2013).

La presente investigación tuvo como objetivo evaluar el efecto de suplementación con probiótico (lactobacillus) en dietas de alfalfa y concentrado sobre parámetros productivos de cuyes mejorados en crecimiento y engorde.

\section{MATERIALES Y MÉTODOS}

\section{Material biológico}

Se emplearon 40 cuyes machos mejorados del tipo 1, de $21 \pm 3$ días de edad, con un peso inicial promedio de 322 g. Fueron identificaron con aretes de aluminio y distribuidos al azar en 20 jaulas. Por cada tratamiento, se tomaron cuatro repeticiones. Cada repetición estuvo conformada por dos animales.
El experimento se realizó en las instalaciones experimentales de cuyes, del Instituto de Investigación en Ganadería y Biotecnología (IGBI) de la Universidad Nacional Toribio Rodríguez de Mendoza de Amazonas, Chachapoyas, región Amazonas y tuvo una duración de sesenta días. Se evaluaron ganancias de peso, consumo de alimento, conversión alimenticia, rendimiento de carcasa y mérito económico.

\section{Tratamientos}

$\mathrm{T}_{1}=$ concentrado + alfalfa verde

$\mathrm{T}_{2}=$ concentrado $+0.2 \%$ probiótico + alfalfa verde $\mathrm{T}_{3}=$ concentrado $+0.3 \%$ probiótico + alfalfa verde $\mathrm{T}_{4}=$ concentrado $+0.4 \%$ probiótico + alfalfa verde $\mathrm{T}_{5}=$ concentrado $+0.5 \%$ probiótico + alfalfa verde

\section{Dietas}

Se formuló un concentrado (tabla 1) de crecimientoengorde. Se realizó un análisis nutricional del concentrado y alfalfa y se calculó el aporte nutricional total (tabla2), indicando suministros de $40 \%$ de concentrado y $60 \%$ de forraje. Se utilizaron los estándares nutricionales reportados por Vergara (2008) y se usó el programa lineal al mínimo costo (MIXIT 2), con 2.80 Mcal ED/Kg y $18 \%$ proteína cruda. La cantidad de concentrado y forraje verde se calculó en función del consumo semanal de materia por peso vivo (tabla 3 ). Del mismo modo se calculó el probiótico, llevando su equivalencia porcentual, por tratamiento, en cantidad de gotas suministradas diariamente en el concentrado (tabla 3 ).

Tabla 1. Composición del concentrado de crecimiento- engorde

\begin{tabular}{lc}
\hline Ingrediente & $\%$ \\
\hline Maíz nacional & 60.10 \\
Torta de soya & 24.95 \\
Polvillo de arroz & 4.00 \\
Afrecho de trigo & 4.00 \\
Melaza de caña & 3.50 \\
Fosfato dicálcico & 2.75 \\
Sal & 0.40 \\
Dl Metionina & 0.15 \\
Hcl Lisina & 0.05 \\
Premezcla & 0.10 \\
\hline Total & 100.00 \\
\hline Ración formulada con MIXIT 2
\end{tabular}


Tabla 2. Análisis nutricional del concentrado y alfalfa

\begin{tabular}{lccc}
\hline Nutriente & $\begin{array}{c}\text { Concentrado } \\
(40 \%)\end{array}$ & $\begin{array}{c}\text { Alfalfa } \\
(60 \%)\end{array}$ & $\begin{array}{c}\text { Aporte } \\
\text { Total }\end{array}$ \\
\hline $\mathrm{MS}^{1}(\%)$ & 85.70 & 88.40 & - \\
$\mathrm{PC}^{2} \quad(\%)$ & 17.10 & 18.00 & 17.64 \\
$\mathrm{FC}^{3} \quad(\%)$ & 2.70 & 22.40 & 14.52 \\
$\mathrm{EE}^{4}(\%)$ & 5.00 & 4.40 & 4.64 \\
$\mathrm{FDN}^{5}(\%)$ & 7.70 & 28.30 & 20.06 \\
$\mathrm{ED}^{6}(\mathrm{Mcal} / \mathrm{kg})$ & 3.10 & 2.63 & 2.82 \\
\hline${ }^{1}$ Materia seca; ${ }^{2}$ Proteina cruda; & ${ }^{3}$ Fibra cruda; \\
etéreo; Extracto
\end{tabular}

Tabla 3. Cantidad diaria, de concentrado, alfalfa y probiótico suministrado según peso semanal del cuy.

\begin{tabular}{|c|c|c|c|c|c|c|c|c|c|c|c|}
\hline \multirow{2}{*}{$\begin{array}{l}\mathrm{N}^{\circ} \\
\text { Sem }\end{array}$} & \multirow{2}{*}{$\begin{array}{l}\text { Peso } \\
\text { Vivo } \\
(\mathrm{g}) \\
\end{array}$} & \multirow{2}{*}{$\begin{array}{l}\text { Consumo } \\
\text { (MS) (g) }\end{array}$} & \multirow{2}{*}{$\begin{array}{c}60 \% \\
\mathrm{MS} \mathrm{FV} \\
\text { (g) }\end{array}$} & \multirow{2}{*}{$\begin{array}{c}40 \% \\
\mathrm{MS} \mathrm{CC} \\
(\mathrm{g})\end{array}$} & \multirow{2}{*}{$\begin{array}{c}\text { FV } \\
(\mathrm{TCO}) \\
(\mathrm{g})\end{array}$} & \multirow{2}{*}{$\begin{array}{c}\mathrm{CC} \\
(\mathrm{TCO}) \\
(\mathrm{g})\end{array}$} & \multicolumn{5}{|c|}{$\begin{array}{l}\text { Número de gotas diarias de } \\
\text { probiótico BIOLAC }\end{array}$} \\
\hline & & & & & & & $\mathrm{T}_{1}$ & $\mathrm{~T}_{2}$ & $\mathrm{~T}_{3}$ & $\mathrm{~T}_{4}$ & $\mathrm{~T}_{5}$ \\
\hline 1 & 370 & 26 & 16 & 10 & 64 & 12 & 0 & 2 & 3 & 4 & 6 \\
\hline 2 & 450 & 32 & 19 & 13 & 77 & 15 & 0 & 2 & 4 & 5 & 7 \\
\hline 3 & 525 & 37 & 22 & 15 & 90 & 17 & 0 & 3 & 4 & 6 & 8 \\
\hline 4 & 600 & 42 & 25 & 17 & 103 & 20 & 0 & 3 & 5 & 7 & 9 \\
\hline 5 & 670 & 47 & 28 & 19 & 115 & 22 & 0 & 4 & 6 & 8 & 10 \\
\hline 6 & 700 & 49 & 29 & 20 & 120 & 23 & 0 & 4 & 6 & 8 & 11 \\
\hline 7 & 770 & 54 & 32 & 22 & 133 & 25 & 0 & 5 & 7 & 9 & 12 \\
\hline 8 & 850 & 60 & 36 & 24 & 146 & 28 & 0 & 5 & 7 & 10 & 13 \\
\hline
\end{tabular}

FV: Forraje verde de alfalfa; CC: Concentrado; MS: Materia seca; TCO: Tal como ofrecido

\section{Parámetros evaluados}

Peso vivo y ganancia de peso

Los pesos se hicieron semanalmente, con una balanza (cavory) digital gramera de capacidad máxima $5 \mathrm{~kg}$. Para ganancia de peso acumulada, se obtuvo de la diferencia del peso a la octava semana de evaluación y el peso inicial. La ganancia de peso, también fue evaluada en forma semanal.

Consumo de alimento: Determinado por diferencia del alimento suministrado en el día y el desperdicio registrado al día siguiente.

Conversión alimenticia: Se calculó estableciendo la relación entre el consumo de alimento y la ganancia de peso.

Rendimiento de carcasa: determinado con el peso de carcasa por 100 sobre el peso vivo. La carcasa incluyo: cabeza, patas, vísceras (corazón, hígado y riñones.

Mérito económico del alimento: Se determinó estableciendo el método del presupuesto parcial a partir de los cambios en los beneficios menos los cambios en los costos.
Diseño estadístico: Para este experimento se empleó el diseño completamente al azar (DCA). Se utilizó el análisis de variancia para determinar existencia de diferencias significativas de los tratamientos $(\alpha=$ 0.05 ) y la prueba estadística de Tukey para comparar las diferencia tratamientos.

\section{RESULTADOS Y DISCUSIÓN}

Se obtuvieron resultados promedios totales por cada parámetro y tratamiento (Tabla 6).

\section{Peso vivo}

Los resultados no evidenciaron diferencias estadísticas significativas entre los tratamientos, por efecto de la suplementación con niveles de probiótico (Tabla 6). Sin embargo, el control obtuvo $12 \%$ de peso menor $\mathrm{T}_{2}$, y en promedio un $5 \%$ menor al $\mathrm{T}_{3} \mathrm{y} \mathrm{T}_{4}$. Siendo inferiores a los pesos reportados por Cerna (1997), esto posiblemente esté relacionado a la mayor cantidad de concentrado que se dio en relación al forraje chala. Asimismo, Rengifo (2005) y Valverde (2006) obtuvieron mayores pesos porque dieron concentrado ad libitum y restringieron al forraje que 
fue brócoli en una proporción del 15 y 10\% de peso vivo en materia seca. Además, Vásquez (2013), Aceijas (2014) y Garibay (2009) obtuvieron mayores pesos finales porque mantuvieron una semana más el periodo de investigación, y estos resultados posiblemente hubieran sido iguales o superiores si se seguía hasta las nueve semanas (Tabla 6).

\section{Ganancia de peso}

Las ganancias de peso no mostraron diferencias significativas $(p>0.05)$ entre los tratamientos evaluados. Sin embargo, se puede apreciar mayores ganancias en $\operatorname{los} \mathrm{T}_{2}, \mathrm{~T}_{3}$ y $\mathrm{T}_{4}$ respecto al control. Siendo el $\mathrm{T}_{2}$ superior en un $24 \%$ al $T_{1}$ (Tabla 6).

La menor ganancia de peso exhibida con la dieta que recibieron el $\mathrm{T}_{1}$ y el $\mathrm{T}_{5}$, podría deberse al ligero menor consumo, trayendo como resultado la menor ingestión de nutrientes tales como la proteína total, la energía digestible y la fibra (Tabla 6). Siendo inferiores a los reportes de Cerna (1997), Tiznado (2013) y Vásquez (2013) con promedios de 16.9,10.2, $11.4 \mathrm{~g}$ /día; posiblemente estas mayores ganancias de peso fue porque la alimentación fue ad livitum y no se restringió como en este estudio. Asimismo, Rengifo (2005), Valverde (2006), Garibay (2009) y Aceijas (2014) obtuvieron mayores ganancias de peso (21.0, 15.5, 13.0 y $10.7 \mathrm{~g}$ /día respectivamente). Estas diferencias de pesos probablemente estén relacionadas a la inclusión de levadura hidrolizada, péptidos y nucleótidos.

\section{Consumo de alimento}

Los consumos no presentaron diferencias significativas entre tratamientos y el control ( $p>0.05)$. Sin embargo numéricamente el $\mathrm{T}_{2}$ y $\mathrm{T}_{3}$ obtuvieron ligeros mayores consumos respecto al control. Camino y Hidalgo (2014) reportaron consumos superiores en cuyes de genotipo Perú (48.2 g/día) y Cieneguillas (49 g/día). Además, inferiores a los consumos reportados por Valverde (2006), Garibay (2009) y Vásquez (2013) debido a la restricción de alimento realizada en esta investigación.

\section{Conversión alimenticia}

No hubo diferencias estadísticas significativas $(\mathrm{p}>0.05)$ en la conversión alimenticia promedio entre los tratamientos; sin embargo los $\mathrm{T}_{2}, \mathrm{~T}_{3}$ y $\mathrm{T}_{4}$ fueron mejores al $\mathrm{T}_{1}$ en un 24, 28 y 29\% menor respectivamente. Esto indicaría más eficiencia en su conversión de alimento a carne. Siendo similares a las conversiones reportadas por Vásquez (2013) y Aceijas (2014) en cuyes ecotipo Cajamarca (6.7 y 6.4 respectivamente) bajo un sistema de alimentación mixta. Pero inferiores a los resultados de Valverde (2006), Garibay (2009), Tiznado (2013) y Camino y Hidalgo (2014) con conversiones de 3.3,3.5, 5.7, y 3.33 respectivamente.

Vásquez y Aceijas obtuvieron conversiones semejantes a esta investigación con niveles de 0.3 y $0.4 \%$ de probiótico, indicando dato más exactos de conversión en un sistema de crianza en alimentación mixta, estos autores trabajaron con forraje alfalfa y concentrado en comparación a otros autores con broza de brócoli, además trabajaron en condiciones ambientales más similares a este experimento, sin embargo estos autores al no restringir en concentrado obtuvieron mayor consumo y mayores ganancias de peso en relación a esta investigación.

Tabla 6: Parámetros productivos de los cuyes mejorados bajo los diferentes tratamientos

\begin{tabular}{|c|c|c|c|c|c|}
\hline \multirow[b]{2}{*}{ Tratamiento } & \multicolumn{5}{|c|}{ Parámetros productivos } \\
\hline & $\begin{array}{l}\text { Peso final } \\
\text { (g) }\end{array}$ & $\begin{array}{l}\text { Ganancia } \\
\text { diaria de } \\
\text { peso }(\mathrm{g})\end{array}$ & $\begin{array}{l}\text { Consumo } \\
\text { diario (g) }\end{array}$ & $\begin{array}{l}\text { Conversión } \\
\text { alimenticia }\end{array}$ & $\begin{array}{l}\text { Rendimiento } \\
\text { carcasa }(\%)\end{array}$ \\
\hline $\mathrm{T}_{1}:$ Control & $r s$ & $7.6 \pm 1.1^{\mathrm{a}}$ & $44.7 \pm 0.2^{a}$ & $9.3 \pm 3.2^{\mathrm{a}}$ & $71.3 \pm 0.8^{\mathrm{a}}$ \\
\hline $\mathrm{T}_{2}: 0.2 \%$ Probiótico & $852 \pm 33.9^{a}$ & $9.4 \pm 1.1^{\mathrm{a}}$ & $45.2 \pm 0.4^{\mathrm{a}}$ & $7.1 \pm 3.2^{\mathrm{a}}$ & $72.6 \pm 1.4^{\mathrm{a}}$ \\
\hline $\mathrm{T}_{3}: 0.3 \%$ Probiótico & $800 \pm 73.9^{a}$ & $8.7 \pm 1.4^{\mathrm{a}}$ & $45.1 \pm 0.7^{\mathrm{a}}$ & $6.7 \pm 1.3^{\mathrm{a}}$ & $71.5 \pm 2.0^{\mathrm{a}}$ \\
\hline $\mathrm{T}_{4}: 0.4 \%$ Probiótico & $804 \pm 44.4^{\mathrm{a}}$ & $8.9 \pm 0.7^{\mathrm{a}}$ & $44.9 \pm 0.5^{\mathrm{a}}$ & $6.6 \pm 1.6^{\mathrm{a}}$ & $70.5 \pm 1.2^{\mathrm{a}}$ \\
\hline $\mathrm{T}_{5}: 0.5 \%$ Probiótico & $752 \pm 52.6^{a}$ & $7.4 \pm 1.0^{\mathrm{a}}$ & $44.7 \pm 0.6^{\mathrm{a}}$ & $9.4 \pm 2.0^{\mathrm{a}}$ & $70.2 \pm 2.7^{\mathrm{a}}$ \\
\hline
\end{tabular}

Letras iguales en la misma fila, no muestran diferencias significativas ( $\mathrm{p}>0.05)$. Los datos corresponden a media y desviación estándar

Estas diferencias de conversiones probablemente estén relacionadas a la proporción de forraje y concentrados que se le brinda a los cuyes, siendo mayor el concentrado respecto al forraje (Tiznado, 2013 y Camino y Hidalgo, 2014), pero en esta investigación se consideró $40 \%$ concentrado y $60 \%$ forraje en MS, usando el mayor recurso 
disponible alfalfa de la zona. Esto diferirá en los costos de producción.

\section{Rendimiento de carcasa}

No se muestran diferencias significativas $(p>0.05)$ entre los tratamientos, sin embargo numéricamente el $\mathrm{T}_{2}$ fue mayor en un $2 \%$ al $\mathrm{T}_{1}$. Al respecto, Rengifo (2005), Valverde (2006) y Camino y Hidalgo (2014) obtuvieron rendimiento de carcasa de $71.9 \%$ y $73.2 \%$ y $73 \%$ respectivamente, siendo similares al de este estudio. Pero superior al reporte de Garibay (2009) que presento un $68.0 \%$ rendimiento de carcasa.

\section{Mérito económico del alimento}

En la Tabla 7, se muestra el efecto de los cinco tratamientos sobre el mérito económico en nuevos soles por cuy, así como también se muestran los precios de los concentrados, alfalfa y del probiótico de bacterias lácticas en nuevos soles por kilogramo de alimento.

Tabla 7. Efecto del probiótico de sobre la retribución económica del alimento.

\begin{tabular}{|c|c|c|c|c|c|}
\hline \multirow{2}{*}{ Parámetros } & \multicolumn{5}{|c|}{ Niveles probiótico de bacterias lácticas } \\
\hline & $0 \%$ & $0.2 \%$ & $0.3 \%$ & $0.40 \%$ & $0.50 \%$ \\
\hline $\mathrm{PF}^{1}(\mathrm{~g})$ & 759 & 852 & 800 & 804 & 752 \\
\hline $\mathrm{PV}^{2}(\mathrm{~S} / . \mathrm{kg})$ & 15.0 & 15.0 & 15.0 & 15.0 & 15.0 \\
\hline IB $^{3}$ (S/. Cuy) & 11.39 & 12.78 & 12.00 & 12.06 & 11.28 \\
\hline \multicolumn{6}{|c|}{ Dieta crecimiento y engorde } \\
\hline $\mathrm{CC}^{4}$ (g/cuy) & 1253 & 1225 & 1218 & 1239 & 1239 \\
\hline $\mathrm{PC}^{5}(\mathrm{~S} / . \mathrm{Kg})$ & 1.60 & 1.60 & 1.60 & 1.60 & 1.60 \\
\hline $\mathrm{CAl}^{6}(\mathrm{~g} /$ cuy $)$ & 5887 & 6076 & 6069 & 5978 & 5929 \\
\hline $\mathrm{PAl}^{7}(\mathrm{~S} / . \mathrm{Kg})$ & 0.80 & 0.80 & 0.80 & 0.80 & 0.80 \\
\hline $\mathrm{PP}^{8}(\mathrm{~S} / . \mathrm{Kg})$ & 15.0 & 15.0 & 15.0 & 15.0 & 15.0 \\
\hline $\mathrm{CP}^{9}$ (g/cuy) & 0.0 & 4.8 & 7.3 & 9.8 & 13.0 \\
\hline $\mathrm{CoA}^{10}(\mathrm{~S} /)$. & 6.71 & 6.89 & 6.91 & 6.91 & 6.92 \\
\hline $\operatorname{MECL}^{11}$ (S/.) & 4.67 & 5.89 & 5.09 & 5.15 & 4.36 \\
\hline $\operatorname{RR}^{12}(\%)$ & 100 & 126 & 109 & 110 & 93 \\
\hline
\end{tabular}

${ }^{1}$ Peso promedio final cuy, ${ }^{2}$ Precio de venta cuy, ${ }^{3}$ Ingreso bruto por cuy, ${ }^{4}$ Consumo concentrado, ${ }^{5}$ Precio concentrado , ${ }^{6}$ Consumo alfalfa, ${ }^{7}$ Precio alfalfa, ${ }^{8}$ Precio probiótico, ${ }^{9} \mathrm{Consumo}$ probiótico, ${ }^{10}$ Costo de alimentación, ${ }^{11}$ Mérito económica por cuy logrado , ${ }^{12}$ Retribución relativa.

El mejor mérito económico por cuy logrado, con la aplicación de $0.2 \%$ probiótico fue en $26 \%$ al $\mathrm{T}_{1}$. Los $\mathrm{T}_{3}$ y $\mathrm{T}_{4}$ también obtuvieron un mejor mérito económico de $9 \%$ y $10 \%$ en relación al control. Siendo similares a los hallazgos de Cerna (1997), Tiznado (2013), Aceijas (2014) quienes obtuvieron méritos económicos de 108, 112 y $109 \%$ respectivamente, respecto a su tratamiento control. Además, el tratamiento con $0.2 \%$ probiótico tuvo mayor mérito económico que estos autores (Tabla 7). Cabe indicar que Cerna (1997) y Tiznado (2013), evaluaron la levadura como prebiótico, proteico y energético y mejoraron en relación a su testigo, similar a esta investigación.

Aceijas (2014) obtuvo un mejor mérito de su dieta mixta (concentrado más alfalfa) en relación a una dieta concentrado, esto nos daría una noción en esta investigación si se hubiera evaluado niveles de probióticos en una dieta mixta o con solo concentrado, posiblemente el uso de recursos disponibles en la zona logre abaratar costos de alimentación.

\section{CONCLUSIONES}

La adición de probiótico de bacterias lácticas, en dieta mixta (alfalfa y concentrado) de cuyes no influye en los parámetros productivos del cuy, como el consumo de alimento, pesos finales, ganancias de peso, conversiones alimenticias y rendimiento de carcasas. Sin embargo en tratamiento 2 con $0.2 \%$ de probiótico tuvo un mayor peso final, ganancia de peso, consumo de alimento y rendimiento de carcasa que el control y los demás tratamientos y una conversión más eficiente que le control, esto es numéricamente.

Los cuyes alimentados con suplemento de $0.2 \%$ de probiótico mostraron un mejor mérito económico respecto a los demás tratamientos. Sin embargo a niveles de $0.5 \%$ de probiótico, los cuyes presentaron un inferior merito respecto a los demás tratamientos. Según los hallazgos de este estudio, se recomienda el uso de probiótico (bacterias acido lácticas) en un rango de $0.2 \%$ hasta $0.4 \%$ en la inclusión de la dieta.

\section{BIBLIOGRAFÍA}

Aceijas, P. (2014). Efecto del tipo de alimento y sexo sobre el comportamiento productivo, características de la carcasa y calidad de la carne del cuy (Cavia porcellus) en la provincia de Cajamarca. Tesis. UNC. Cajamarca. Perú.

Beasley, S. S., Takala, T. M., Reunanen, J., Apajalahti, J., \& Saris, P. E. J. (2004). Characterization and electrotransformation of Lactobacillus crispatus isolated from chicken crop and intestine. Poultry science, 83(1), 4548.

Bocourt, R., Savón, L., \& Díaz, J. (2002). Efecto de la actividad probiótica de Lactobacillus rhamnosus en indicadores fisiológicos, productivos y de salud de cerdos jóvenes. XVIII Congreso Panamericano de Ciencias Veterinarias. Palacio de las Convenciones. La Habana, Cuba (p. 135).

Bourliux, P., Koletzko, B., Guarner, F., Braesco, V. 
(2002). The intestine and microflora are partners in protection of the host. Am J Clin Nutr 78, 675-683.

Cerna, M. (1997). Evaluación de cuatro niveles de residuo de cervecería seco en el crecimiento y engorde de cuyes. Tesis para optar el grado de ingeniero zootecnista UNALM. Lima. Perú.

García, Y., López, A., Boucourt, R., Elías, A., \& Dihigo, L. E. (2002). Efecto del tratamiento térmico en un hidrolizado enzimático de crema de levadura Saccharomyces cerevisiaeen los niveles de colesterol en pollos de ceba. Revista Cubana de Ciencia Agrícola, 36(4), 361-365.

Garibay, M. (2009). Evaluación de tres programas de alimentación mixta en el comportamiento productivo de cuyes en crecimiento. Tesis para optar el grado de ingeniero zootecnista UNALM. Lima. Perú

Gutiérrez Ramírez, L. A., Montoya, O. I., \& Vélez Zea, J. M. (2013). Probiotics: an alternative for cleaner production and a possible replacement of the antibiotics as growth promoters in animal feeding. Producción+ limpia, 8(1), 135-146.

Mountzouris, K. C., Tsirtsikos, P., Kalamara, E., Nitsch, S., Schatzmayr, G., \& Fegeros, K. (2007). Evaluation of the efficacy of a probiotic containing Lactobacillus, Bifidobacterium, Enterococcus, and Pediococcus strains in promoting broiler performance and modulating cecal microflora composition and metabolic activities. Poultry Science, 86(2), 309-317.

Pascual, M., Badiola, M., Monfor, J., \& Garriga, M. (1999). Lactobacillus salivarius prevents Salmonella enteritidis colonization in chickens.Appl. Environm. Microbiol, 65, 4981-4986.

Pérez, M. Laurencio, M. Piad, R. Milián, G y Rondón A. (2002). Evaluación de la actividad probiótica de un producto de exclusión competitiva sobre indicadores microbiológicos en el ciego de pollos de ceba. Rev. Cubana de Ciencia Avícola. 26, 29-35.

Rengifo, G. (2005). Evaluación de alimento balanceado peletizado y en harina con suministro de forraje en cuyes (cavia porcellus) mejorados. Tesis para optar el grado de ingeniero zootecnista UNALM. Lima. Perú.

Tiznado, S. (2013). Efecto de las levaduras hidrolizadas (Saccharomyces cerevisiae) en alimentación de cuyes sobre indicadores productivos de crecimiento. Tesis para optar el grado de ingeniero zootecnista UNC. Cajamarca. Perú.

Valverde, C. (2006). Evaluación de cuatro áreas de crianza por animal en el crecimiento de cuyes (cavia porcellus) mejorados. Tesis para optar el grado de ingeniero zootecnista UNALM. Lima. Perú.

Van Soest, P. (1983). The lower tract. En: Van Soest P, ed. Nutritional ecology of the ruminant. USA: Cornell University. p 195-210.

Vásquez, C. (2013). Inclusión de péptidos y nucleótidos en el alimento concentrado de cuyes en crecimiento bajo sistema de alimentación mixta. Tesis para optar el grado de ingeniero zootecnista UNC. Cajamarca. Perú.

Vergara, V. (2008). Simposio Avances sobre producción de cuyes en el Perú. XXXI Reunión científica anual de la Asociación Peruana de Producción Animal 2008.36 p.

Leandro, G., \& Victoria, M. (2012). Caracterización de la actividad de las enzimas hidrolíticas localizadas en la región cecal de cuyes (cavia porcellus).

Saavedra Sierra, D. M. (2018). Forraje verde hidropónico de tres variedades de cebada (hordeum vulgare) en la dieta de cuyes (cavia porcellus) en recría. Tesis para optar el grado de Médico veterinario Universidad Nacional Micaela Bastidas de Apurímac, Abancay, Perú. $81 \mathrm{p}$.

Huertas, R. A. P. (2010). Bacterias ácido lacticas: papel funcional en los alimentos. Ingresar a la revista, 8(1), 93-105.

Mejia, M., \& Vanessa, P. (2011). Influencia de la inclusión de pronutrientes y prebiótico en los indicadores productivos de pollos de engorde en la etapa de arranque. Tesis para optar el grado de doctor, Universidad de El Salvador. San Salvador. 70 p.

Camino, J., \& Hidalgo, V. (2014). Evaluación de dos genotipos de cuyes (Cavia porcellus) alimentados con concentrado y exclusión de forraje verde. Revista de Investigaciones Veterinarias del Perú, 25(2), 190-197. 